\title{
Pielonefritis xantogranulomatosa: Estudio retrospectivo de 8 casos
}

Dres. Diego Miguel Soriano M, Juan Manuel Sanchís G, María del Carmen Gómez M, Sandra Emilia González I, Julio Palmero D.

1. Servicio de Radiodiagnóstico, Hospital Clínico Universitario de Valencia. España.

2. Servicio de Radiodiagnóstico, Hospital Clínico Universitario de Valencia. España.

3. Servicio de Anatomía Patológica. Hospital Clínico Universitario de Valencia. España.

4. Servicio de Radiodiagnóstico del Hospital de la Ribera. Alzira. Valencia. España.

5. Servicio de Radiodiagnóstico, Hospital Clínico Universitario de Valencia. España.

\section{Xanthogranulomatous pyelonephritis: a retrospective study of 8 cases}

Abstract: We have revised the clinical, radiological and pathological characteristics of Xanthogranulomatous pyelonephritis (XGP), together with the evolution of the disease and a literature review of the same. A retrospective study was performed at our center, obtaining a sample of 8 pathologically confirmed cases which we used to describe the clinical and diagnostic features. Of the 8 cases ( 1 male and 7 female), 6 of them presented complicated renal colic, one presented asthenia and weight loss and the other case was an incidental finding. The initial diagnosis was achieved with ultrasonography in 2 of the cases and with $C T$ in all of the cases, all being confirmed by a histopathological study.

Keywords: Calculi (lithiasis), Multidetector CT, Pyelonephritis, Xanthogranuloma.

Resumen: Hemos revisado las características clínicas, radiológicas y patológicas de la pielonefritis xantogranulomatosa junto con la evolución de la enfermedad y una revisión bibliográfica de la misma. Se realiza un estudio retrospectivo en nuestro centro, obteniendo una muestra de 8 casos confirmados por anatomía patológica a través de los que describimos las características clínicas y diagnósticas. De los 8 casos (1 varón y 7 mujeres), 6 de ellos debutaron como cólico renal complicado, 1 se presentó con astenia y pérdida de peso y el otro caso fue un hallazgo incidental. El diagnóstico inicial se realizó mediante ecografía en 2 casos y TC en todos los casos, siendo confirmados todos mediante estudio histopatológico. Palabras clave: Litiasis, Pielonefritis, TC multidetector, Xantogranuloma.

Soriano M, et al. Pielonefritis xantogranulomatosa: Estudio retrospectivo de 8 casos. Rev Radiol 2013; 19(3): $97-102$. Correspondencia: Dr. Miguel Soriano M. / disome72@ hotmail.com

Trabajo recibido el 09 de junio de 2013, aceptado para publicación el 30 de agosto de 2013.

\section{Introducción}

La pielonefritis xantogranulomatosa (PNX) fue descrita por primera vez en 1916 por Schlagenhaufer(4). Se trata de una forma infrecuente de infección crónica renal que afecta principalmente de forma difusa y en escasas ocasiones con distribución focal ${ }^{(1-3)}$, siendo necesario en estos casos realizar el diagnóstico diferencial con las neoplasias renales.

Se ha asociado a distintos mecanismos etiopatogénicos como son la obstrucción crónica del tracto urinario, hemorragia, insuficiencia arterial, obstrucción venosa, alteración del sistema inmune, alteración del metabolismo de los lípidos, hiperparatiroidismo y diabetes mellitus ${ }^{(2,3,5-7)}$.

La entidad es más prevalente entre la quinta y séptima década de la vida y más frecuente en mujeres (70\%), a excepción de aquellas que se producen a edades tempranas que no presentan diferencia entre $\operatorname{sexos}^{(2,3)}$.

La clínica es inespecífica y de evolución tórpida, destacando síntomas como astenia, pérdida de peso, dolor en fosa renal y febrícula recurrente. En la ex- 
ploración se puede palpar una masa abdominal en los casos más evolucionados y analíticamente puede presentar leucocitosis, anemia, elevación de la velocidad de sedimentación globular, piuria y proteinuria. Aunque los cultivos de orina pueden ser estériles, es posible llegar a aislar diversos microorganismos como la Escherichia Colli, Proteus mirabilis, Klebsiella y Pseudomonas ${ }^{(8,9)}$.

Existen diversas técnicas de imagen útiles para el diagnóstico inicial, destacando la radiografía abdominal, urografía intravenosa, pielografía retrógrada y angiografía, aunque en la práctica se utiliza la ecografía y la TC por tener mayor sensibilidad y especificidad $^{(10-17)}$.

La PNX se caracteriza macroscópicamente por una afectación unilateral en la que destaca un riñón agrandado, con hidronefrosis, litiasis, fibrosis y formación de masas redondeadas y parduzcas que pueden extenderse hacia la pelvis renal y espacio perirrenal e incluso formar abscesos y fístulas en los casos más evolucionados ${ }^{(18-20)}$.

El diagnóstico de confirmación es histológico, caracterizado por una mala diferenciación córticomedular al corte y que a nivel microscópico muestra una infiltración inflamatoria aguda de predominio polimorfonuclear con macrófagos cargados de lípidos intracitoplasmaticos y células gigantes multinucleadas, imagen histopatológica característica que dan nombre a la entidad ${ }^{(18-20)}$.

El tratamiento de elección es la nefrectomía tras la que existe buen pronóstico, no observándose recidivas. La principal, aunque infrecuente complicación es la sepsis postquirúrgica tras la manipulación de un foco infeccioso ${ }^{(5,6,21)}$.

Presentamos 8 casos destacando las características radiológicas, además de los aspectos clínicos y evolutivos de una enfermedad infrecuente que hasta la fecha no ha sido tratada en muchas publicaciones.

\section{Material y métodos}

Se dispone de 14 casos recogidos durante un período de 5 años que fueron diagnosticados inicialmente como PNX mediante diversas técnicas radiológicas. De los 14 casos excluimos 6 por no tener confirmación histopatológica, quedando 8 casos. El estudio contó con el consentimiento informado de todos los participantes.

Desde el punto de vista epidemiológico describimos la edad de afectación, el sexo y el riñón lesionado. En cuanto a la clínica describimos la forma de presentación y los datos analíticos más relevantes.

Destacamos las distintas técnicas de radiodiagnóstico empleadas en el diagnóstico inicial de la enfermedad, resaltando aquellas características que son comunes o excepcionales en la manifestación de la entidad.

El examen ecográfico se realizó con una plata- forma Siemens Sonoline Antares, (Siemens, Lyons, Colorado) con sonda convexa 4-1 MHz.

Las TC abdominopélvicas se realizaron con un equipo Toshiba (Toshiba Medical Systems Corporation. Asian. Otawa-Shi, Tochig), los tres primeros con un equipo de 16 detectores y los restantes cinco con equipo de 80 detectores con las siguientes características técnicas: TC abdomino-pélvica desde apéndice xifoides hasta sínfisis del púbica, kVp 110 y mAs automático dependiendo del escanograma. Flujo de contraste de $2,5-3 \mathrm{ml} / \mathrm{s}$ y un volumen de contraste de $1 \mathrm{ml} / \mathrm{Kg} /$ peso. Se realizó protocolo en tres fases que incluía una primera fase sin contraste, otra con administración de contraste intravenoso y adquisición de imágenes en fase arterial realizada a los 25 segundos y una última fase venosa a los 65 segundos desde la administración del contraste intravenoso.

Las imágenes fueron evaluadas con el programa Impax versión 6.4.0.4551 de Agfa Healthcare (Agfa Healthcare NV-Corporate Headquarters, Mortsel, Belgium).

Se describe el tratamiento que reciben los pacientes y las características histopatológicas de los casos.

\section{Resultados}

De los 8 casos de PNX, 7 fueron mujeres (87,5\%) y uno varón (12,5\%). La edad media de afectación fue 67 años (rango 42-88 años). El riñón izquierdo se afectó en 4 casos (50\%) y el derecho en los otros cuatro (50\%).

Las manifestaciones clínicas fueron muy variables, desde un caso asintomático (12,5\%), hallazgo incidental tras realizar una TC abdomino pélvica para el estadiaje de un carcinoma rectal, hasta clínica muy manifiesta de cólico nefrítico en 6 casos $(75 \%)$, mientras que en el caso restante predominó clínica de astenia y pérdida de peso $(12,5 \%)$.

La analítica se caracterizó en todos los casos por elevación de la creatinina por insuficiencia renal obstructiva en relación a litiasis renal. Otro dato común en cuatro casos $(50 \%)$ fue la leucocitosis. En 4 pacientes el urocultico fue positivo (50\%); en tres frente a Escherichia Coli $(37,5 \%)$ y en otro para Pseudomona Aeruginosa (12,5\%). En los otros cuatro pacientes el urinocultivo fue negativo (50\%).

En los 6 casos (75\%) que cursaron con cólico nefrítico, se realizó primeramente radiografía abdominal y posteriormente ecografía abdominal para valorar la dilatación del sistema excretor. En los otros dos pacientes $(25 \%)$ la primera prueba de imagen que se realizó fue la TC.

En los 6 casos con clínica de cólico nefrítico (75\%) la radiografía de abdomen mostró agrandamiento de la silueta renal. En dos de estos pacientes se observó litiasis localizada en el sistema excretor y en uno la litiasis presentaba un aspecto coraliforme. 
Con la ecografía se observó en el riñón afectado un agrandamiento del tamaño cráneo-caudal por encima de los límites de la normalidad (entre 12 y $18 \mathrm{~cm}$ ) junto con marcada dilatación del sistema pielocalicial. En cuatro de los seis casos estudiados por cólico nefrítico se identificaron estructuras hiperecogénicas con sombra acústica posterior en relación con litiasis del sistema pielocalicial. La cortical presentaba marcada heterogeneidad con áreas hiperecogénicas y otras hipoecogénicas (Figura 1).

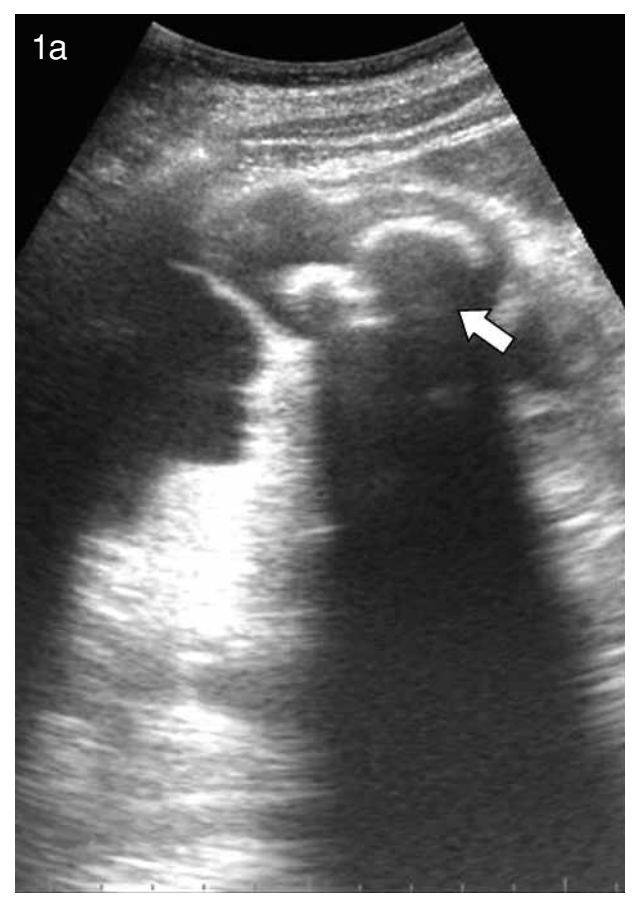

Figura 1. Ecografía abdominal en modo B. a) Litiasis coraliforme (flecha). b) Lesión nodular con aumento de tamaño y desestructuración renal con marcada heterogeneidad, observando áreas hipoecogénicas (asterisco) e hiperecogénicas (cruz).

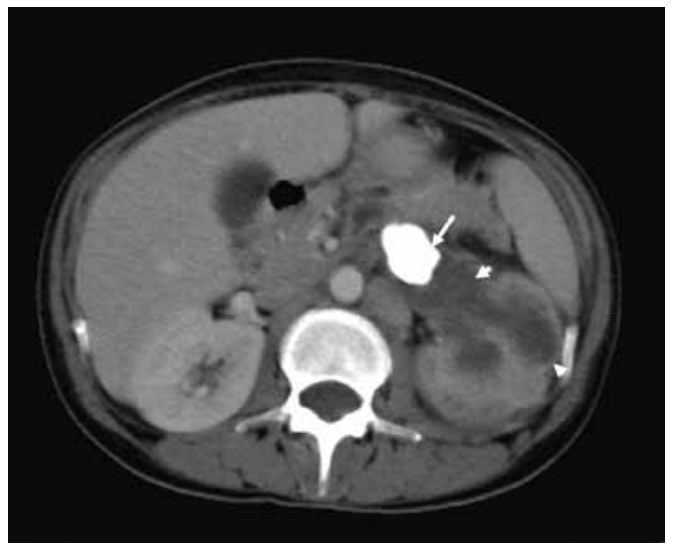

Figura 2. TC abdomino pélvica con administración de contraste intravenoso, plano axial. Se observa agrandamiento de silueta renal, litiasis impactada en pelvis renal (flecha larga), dilatación del sistema pielocalicial (flecha corta) y adelgazamiento de cortical renal (punta de flecha).
Mediante TC en 6 casos se observó aumento del tamaño renal, cortical adelgazada con captación de contraste y dilatación de los distintos grupos caliciales. Además se detectó la presencia de litiasis impactada en pelvis renal en 4 casos y en dos casos localizada en tercio medio y distal (Figura 2). En todos los pacientes se identificó trabeculación de la grasa perirrenal y engrosamiento de la fascia pararrenal por cambios inflamatorios (Figura 3). En dos casos no se detectó litiasis en vía excretora apareciendo únicamente los últimos cambios descritos.
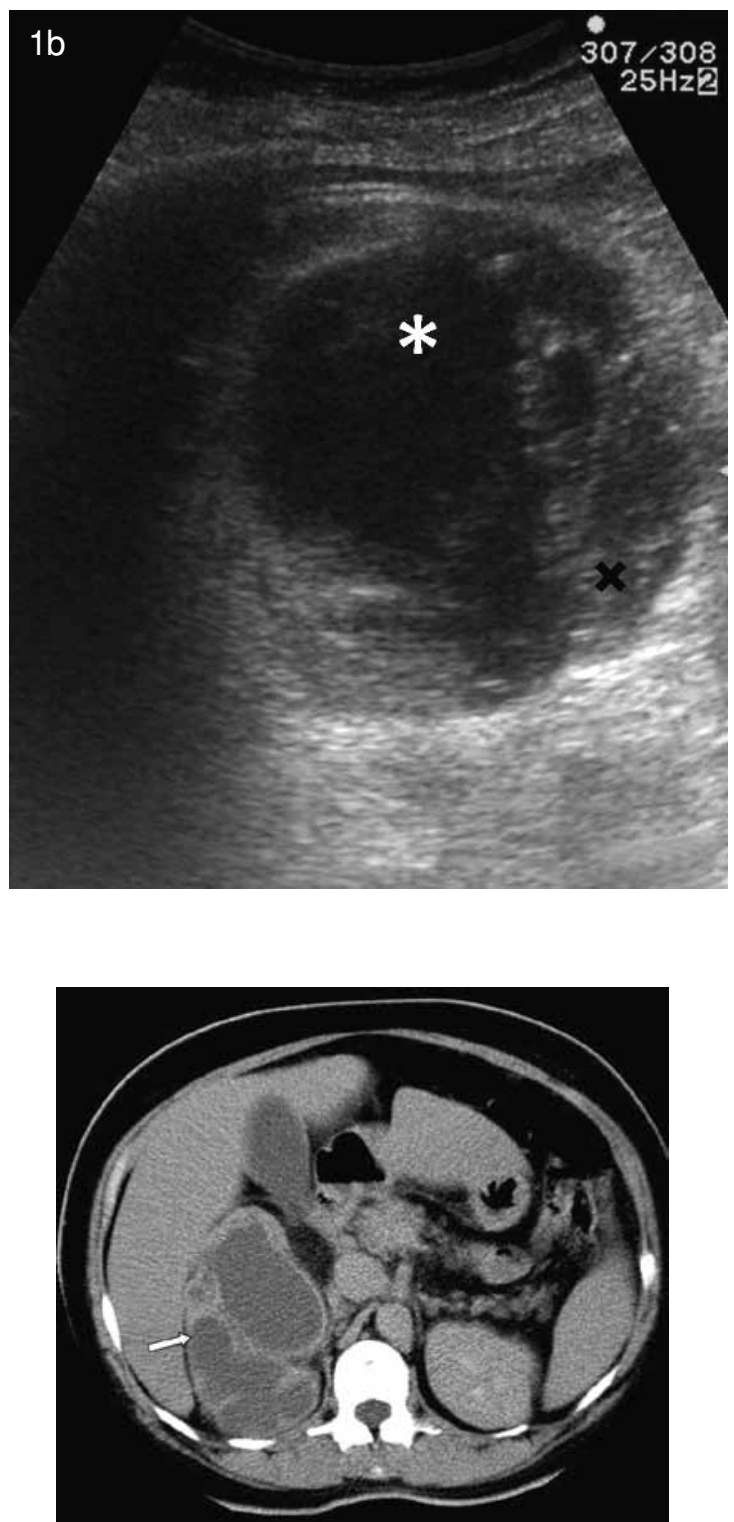

Figura 3. TC abdomino pélvica con administración de contraste intravenoso, plano axial donde se observaba agrandamiento de silueta renal junto con nodularidad (no mostrado) y presencia de colecciones organizadas en parénquima renal derecho (flecha), mostrando el signo radiológico característico de "pata de oso" que representa la sustitución del parénquima renal por áreas necróticas y xantomatosas con realce de cortical renal tras administración de contraste intravenoso. 
En todos los pacientes se practicó una nefrectomía radical tras la cual no se produjeron complicaciones postquirúrgicas inmediatas ni tardías.

Las piezas quirúrgicas fueron estudiadas en anatomía patológica confirmándose el diagnóstico de PNX. Macroscópicamente todos los riñones mostraban un aumento de tamaño (el mayor de ellos de $20 \mathrm{~cm}$ de longitud cráneo-caudal), además de presentar hidronefrosis marcada junto con una mala delimitación córtico-medular. El tejido perirrenal se caracterizaba por encontrarse firmemente adherido al parénquima renal y por presentar tractos fibrosos de coloración blanquecina. En el estudio histopatológico se observó la presencia de numerosa celularidad inflamatoria mixta constituida por linfocitos y polimorfonucleares con predominio de estos últimos, acompañados de células plasmáticas, macrófagos espumosos y células gigantes multinucleadas (Figuras 4,5).
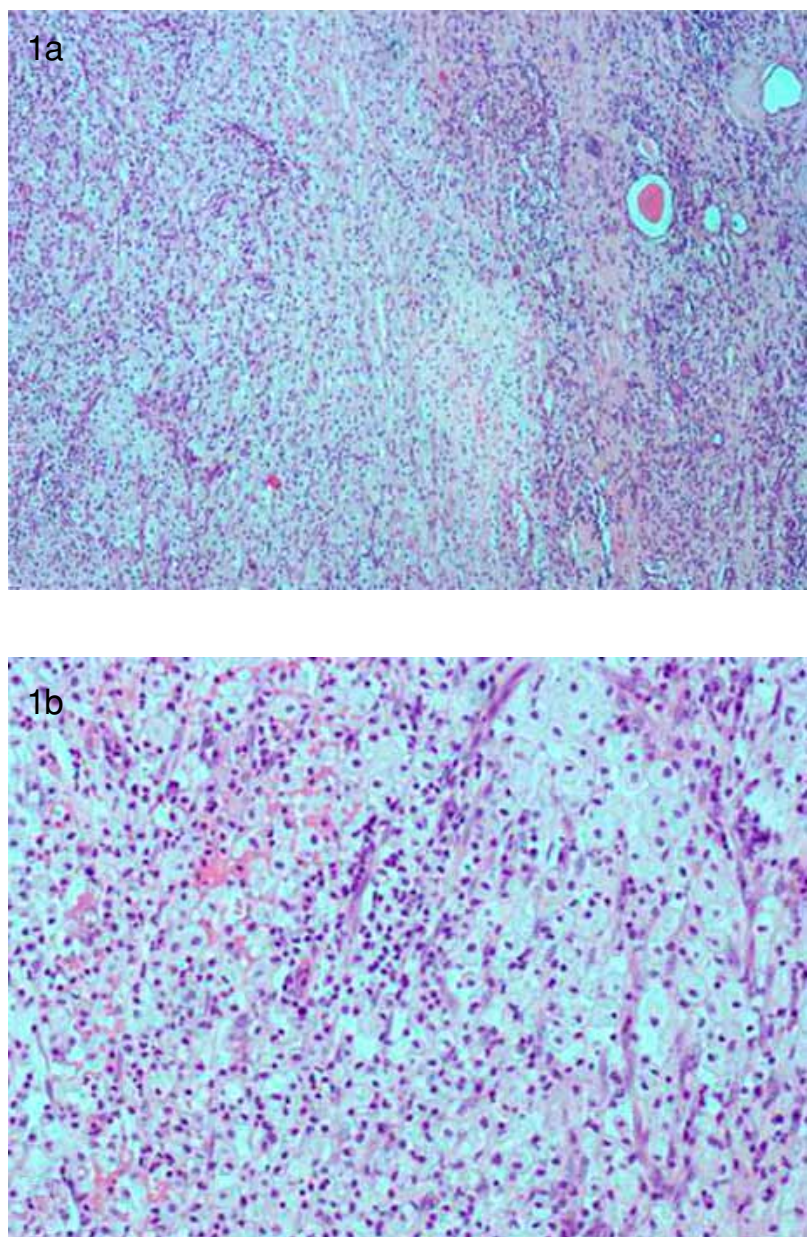

Figura 4. a) Pequeño aumento la lesión, se caracteriza por un infiltrado inflamatorio mixto con fibrosis del parénquima adyacente (hematoxilina-eosina, 40x). b) El infiltrado inflamatorio muestra un predominio de histiocitos xantomatosos de núcleos redondeados y regulares junto a polimorfonucleares y linfocitos (hematoxilina-eosina, 100x).

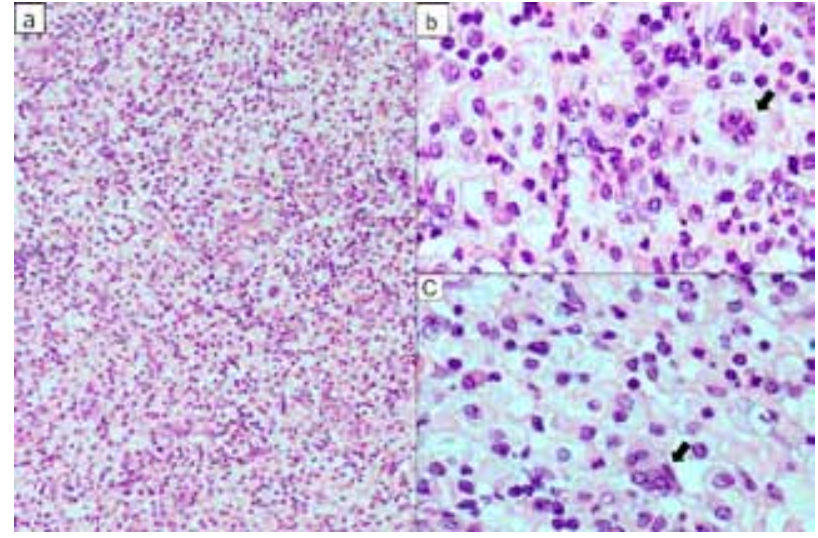

Figura 5. a) El infiltrado inflamatorio está compuesto de un número variable de histiocitos xantomatosos con citoplasma espumoso, neutrófilos y linfocitos que forman agregados (hematoxilina-eosina, 100x). b y c) Detalles de la imagen anterior donde se observa la presencia de células gigantes multinucleadas (flecha) (hematoxilina-eosina, 200x).

\section{Discusión}

Desde la primera descripción de esta enfermedad por Schlagenhaufer en $1916^{(4)}$, la PNX continúa siendo una enfermedad con muy baja incidencia en la población, representando menos del $1 \%$ de las pielonefritis crónicas. Existen pocas publicaciones sobre esta entidad hasta la fecha y menos aún desde la perspectiva radiológica.

En nuestro grupo de pacientes la enfermedad apareció más frecuentemente en mujeres (7:1), con una media de edad de 58 años, afectación unilateral y asociada a litiasis en todos los casos, con una evolución clínica de tipo crónico y síntomas de tipo cólico nefrítico, al igual que la mayoría de las publicaciones realizadas hasta la fecha ${ }^{(22,23)}$. También se ha descrito la presentación como síndrome constitucional con pérdida de peso y astenia ${ }^{(5,6,12,13,23)}$ como sucedió en uno de nuestros pacientes. Otra forma de presentación es la palpación de una masa abdominal en la exploración ${ }^{(6,22,23)}$ situación que no se dio en ninguno de nuestros casos. Con menor frecuencia la enfermedad puede ser detectada como hallazgo incidental al realizar estudios por otra causa ${ }^{(23)} \mathrm{como}$ ocurrió en uno de nuestros casos.

Al igual que en la mayoría de los casos publicados hasta la fecha, nuestros pacientes presentaron analíticamente leucocitosis y elevación de los niveles de creatinina ${ }^{(5,6,23)}$. Por el contrario no se observaron otras alteraciones como la anemia, elevación de la fosfatasa alcalina y de transaminasas descritas en diversos estudios ${ }^{(1,5,6,22,23)}$.

El urocultivo fue positivo en la mitad de los casos $(50 \%)$. Este hallazgo también fue observado por Malek y Elder ${ }^{(1,8,9)}$. Los cultivos negativos se explican porque estos pacientes presentan verdaderos abscesos renales encapsulados y obstrucción de la 
vía urinaria por litiasis, que aíslan los gérmenes de la vía excretora ${ }^{(1,8,9)}$.

Se han descrito diversos mecanismos etiopatogénicos entre los que se encuentra la obstrucción crónica del tracto urinario, hemorragia, insuficiencia arterial, obstrucción venosa, alteración del sistema inmune, alteración del metabolismo lipídico, hiperparatiroidismo y diabetes mellitus. En la literatura se ha descrito la presencia de diabetes mellitus en las distintas series que oscila entre $5-40 \%{ }^{(6)}$. En nuestra serie dos pacientes padecían esta enfermedad (25\%). Por el contrario otros autores no han descrito asociación con esta entidad ${ }^{(7)}$. De los otros factores etiopatogénicos descritos se encontró asociación con obstrucción crónica de la vía excretora en los otros seis pacientes $(75 \%)$.

Los hallazgos radiológicos más destacables fueron agrandamiento renal, y litiasis coincidiendo con otras publicaciones ${ }^{(10-16)}$. La radiografía simple de abdomen permite visualizar litiasis, generalmente coraliforme y agrandamiento de la silueta renal ${ }^{(18-20)}$ como ocurrió en uno de nuestros casos. La ecografía permite demostrar la desestructuración del parénquima, con patrón de hidronefrosis avanzada Figura $1^{(11-19)}$ al igual que sucedió en seis de nuestros casos. La TC puede mostrar agrandamiento renal, presencia de litiasis, dilatación de vía excretora, adelgazamiento cortical, presencia de colecciones peri o pararrenales, cambios inflamatorios de la grasa peri o pararrenal o incluso trayectos fistulosos a piel (10,11,14-17). Se ha descrito el signo de la "pata de oso" que representa la sustitución del parénquima renal por áreas de necrosis o colecciones xantomatosas, imitando una dilatación calicial y realce periférico tras administrar contraste intravenoso Figura $3^{(16)}$. En nuestros pacientes la TC mostró agrandamiento renal, adelgazamiento cortical, dilatación de la vía excretora urinaria y cambios inflamatorios en tejido adyacente, acorde con lo publicado por otros autores Figuras 2, $3^{(10,11,14-17)}$.

El diagnóstico diferencial se debe hacer con tumoraciones renales mediante el estudio anatomopatológico que muestra macroscópicamente un riñón desestructurado, aumentado de tamaño con pobre delimitación córtico-medular y sustitución de su apariencia habitual por un tejido amarillento parduzco de aspecto adiposo, además de la presencia de cavidades quísticas ${ }^{(19,20)}$. Microscópicamente existe infiltración inflamatoria difusa (pielonefritis crónica) caracterizada por polimorfonucleares, linfocitos, células plasmáticas, histiocitos cargados de lípidos denominados células espumosas, y células gigantes multinucleadas ${ }^{(19,20)}$. Estas células espumosas son la que le dan el apellido de xantogranulomatosa a esta enfermedad.

Los tumores renales asociados a PNX, son infrecuentes. Ballesteros ${ }^{(21)}$, menciona en su revisión el $1.7 \%$, siendo los adenocarcinomas renales los más frecuentes seguidos por los uroteliales con una incidencia muy inferior. Los tumores son prácticamente de imposible sospecha en el preoperatorio, ya que están enmascarados por este tipo de pielonefritis requiriendo de un diagnóstico histopatológico. Estos tumores se asocian a urolitiasis de larga evolución e infección urinaria crónica, como factores predisponentes $^{(23)}$. En nuestra serie de casos no se observaron lesiones neoplásicas tras el estudio histopatológico.

En las distintas publicaciones la mortalidad fue entre el 10 y $24 \%$ asociándose en la mayoría de casos a sepsis postquirúrgica ${ }^{(1,6,11,13,21)}$. Al finalizar la revisión de este tema, ninguno de los pacientes de nuestra serie había fallecido.

El número de casos presentado de esta infrecuente entidad que ha sido objeto de estudio hasta la fecha en escasas publicaciones, es uno de los aspectos más positivos de este trabajo, por el contrario, el pequeño tamaño de la muestra limita la potencia estadística de nuestros resultados.

En conclusión, la PNX es una forma infrecuente de pielonefritis crónica que presenta fuerte asociación con litiasis renal, hidronefrosis obstructiva y caracterizada por una clínica variable, desde formas asintomáticas a cuadros clínicos constitucionales y cólicos nefríticos. Desde el punto de vista diagnóstico las diversas técnicas radiológicas nos aproximan al diagnóstico inicial siendo imprescindible la confirmación histopatológica de la enfermedad. El tratamiento de elección es la nefrectomía. La mortalidad se asocia en la mayoría de casos a complicaciones postquirúrgicas por sepsis.

\section{Bibliografía}

1. Malek RS, Elder JS. Xanthogranulomatous pyelonephritis: a critical analysis of 26 cases and of the literature. J Urol 1978; 119(5): 589-593.

2. Malek RS, Greene LF, De Weerd JH, Farrow GM. Xanthogranulomatous pyelonephritis. Br J Urol 1972; 44: 296-308.

3. Anhalt MA, Cawood CD, Scott R. Xanthogranulomatous pyelonephritis: comprehensive review with report of four additional cases. J Urol 1971; 105: 10-17.

4 Schlagenhaufer F. Uber eigentumliche staphylomykosen der nieven und des pararenalen bindegewebes. Frankfurt Z Pathol 1916; 19: 139-148.

5. Korkes F, Favoretto RL, Broglio M y cols. Xanthogranulomatous pyelonephritis: clinical experience with 41 cases. Urology 2008; 71: 178-180.

6. Sobel J. Infecciones urinarias. Mandell GL, Benett JE, Dolin R. .Principios y prácticas en enfermedades infecciosas. 5르. Editorial Panamericana 2002. pp. 60.

7. Berrah-Bennaceur M, Farad AB. La pyelonéphrite santo-granulomateuse. Approche ëtiopathogénigue á propos de duex cas. Sem Hop Paris 1999; 75: 13621367.

8. Leoni AF, Luque A, Sambuelli R, Valverde J. Pielonefritis xantogranulomatosa asociada a flora polimicrobiana. Rev Pan Infec 2004; 6: 23-27. 
9. Watt I, Kristesen IB. Xanthogranulomatous pyelonephritis. Arch Path Microbial Scand Sect A 1980; 89: 89.

10. Alam A, Chander BN. Xanthogranulomatous pyelonephritis: Diagnosis using Computed Tomography. MJAFI 2004; 6: 86-88.

11. Rajesh A, Jakanani G, Mayer N, Mulcahy K. Computed Tomography Findings in Xanthogranulomatous Pyelonephritis. J Clin Imaging Sci 2011; 1: 45.

12. Hartman DS, Davis CJ, Goldman SM, Ibister SS, Sanders R. Xanthogranulomatous pyelonephritis: sonographicpathologic correlation of 16 cases. J Ultrasoun Med 1984; 3: 481-488.

13. Kim J. Ultrasonographic features of focal xanthogranulomatous pyelonephritis. J Ultrasound Med 2004 Mar; 23(3): 409-416.

14. Zorzos I, Moutzouris V, Korakianitis G, Katsou G. Analysis of 39 cases of Xanthogranulomatous pyelonephritis with emphasis on CT and findings. Disponible en: www. ecr.or/t/ECRO2/sciprg/abs/pc/0378.htm; 2000.

15. Craig WD, Wagner BJ, Travis MD. Pyelonephritis: radiologic-pathologic review. Radiographics. 2008; 28: 255-277.

16. Bernstein T,Cabrera R, Barbieri M, Soffla P. El signo la "pata de oso" en TC. Rev Chil Radiol 2011; 17(1): 44-46.

17. Loffroy R, Guiu B, Watfa J, Michel F, Cercueil JP, Krausé $\mathrm{D}$. Xanthogranulomatous pyelonephritis in adults: clinical and radiological findings in diffuse and focal forms. Clin Radiol 2007 Sep; 62(9): 884-890.

18. Saeed SM, FineG. Xanthogranulomatous pyelonephritis. Am J Clin Pathol 1963; 39: 616-625.

19. Hammond NA, Nikolaidis P, Miller FH. Infectious and inflammatory diseases of the kidney. Radiol Clin North Am 2012 Mar; 50(2): 259-270.

20. McDonald GSA. Xanthogranulomatous pyelonephritis. J Pathol 1981; 133: 203-213.

21. Rosoff JS, Raman JD, Del Pizzo JJ. Feasibility of laparoscopic approach in management of Xanthogranulomatous pyelonephritis. Urology 2006; 68: 711-714.

22. Ballesteros Sampol J. Inusuales formas clínicas de presentación y asociaciones patológicas raras de la pielonefritis xantogranulomatosa. Arch Esp Urol 2002; 55(2): 119-130.

23. Tiguert R, Gheiler EL, Yousif R, Tefilli MV, Mills K, Grignon DJ et al. Xanthogranulomatous pyelonephritispresenting as a Renal tumor whith vena cava thrombus. J Urol 1998; 160: 117-118.

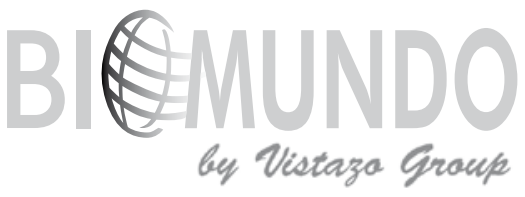

UN MUNDO DE SOLUCIONES EN IMAGEN
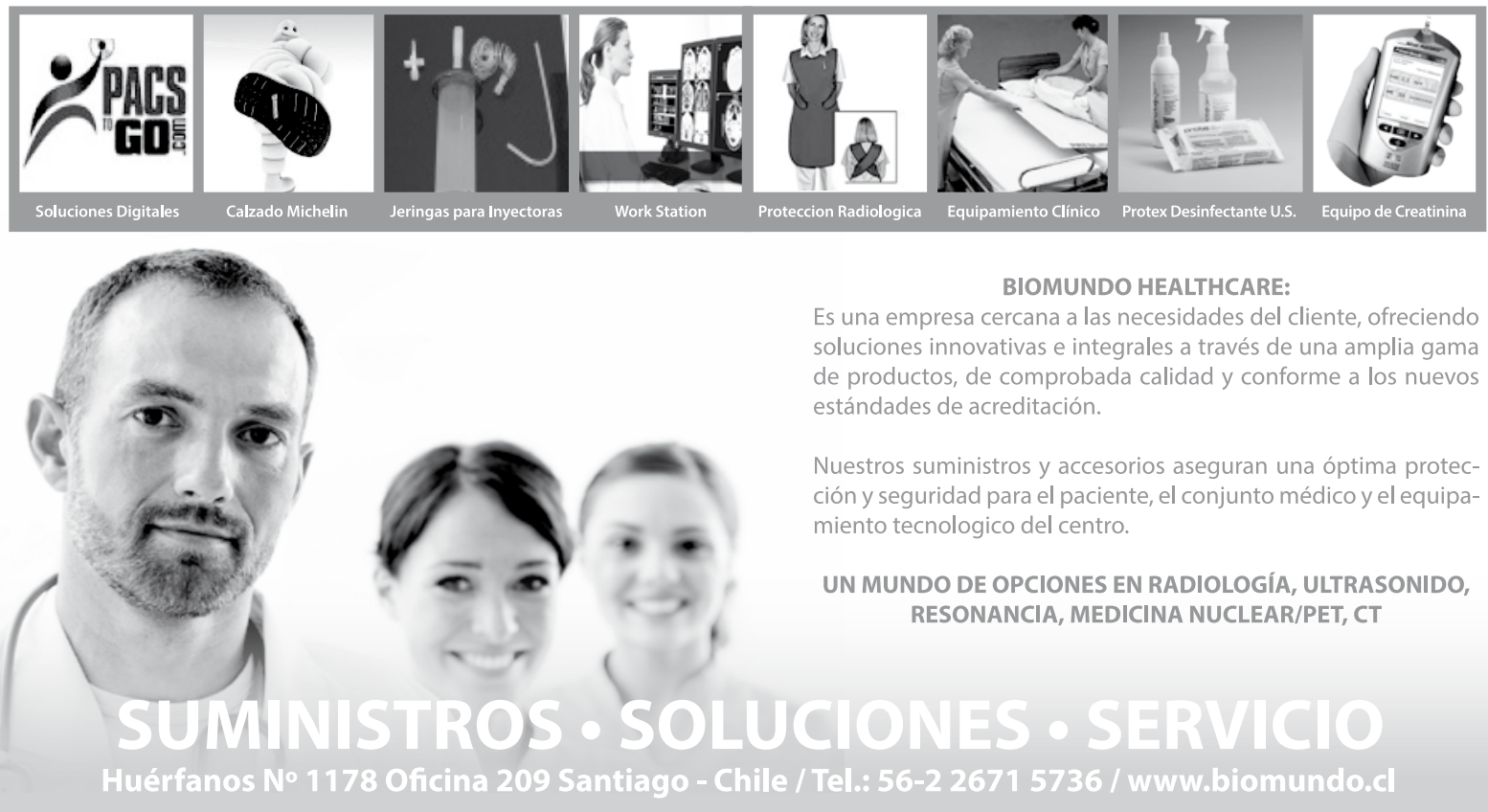